\title{
On the spectral properties of translation operators in one-dimensional tubes
}

\author{
by WojcIEch HyB (Warszawa)
}

\begin{abstract}
We study the spectral properties of some group of unitary operators in the Hilbert space of square Lebesgue integrable holomorphic functions on a one-dimensional tube (see formula (1)). Applying the Genchev transform ([2], [5]) we prove that this group has continuous simple spectrum (Theorem 4) and that the projection-valued measure for this group has a very explicit form (Theorem 5).
\end{abstract}

Let $D=\{z \in \mathbb{C}: \operatorname{Im} z \in J\}$ be a tube over an open interval $J \subset \mathbb{R}, J \neq$ R. Let $L^{2} H(D)$ be the Hilbert space of all functions which are holomorphic and square integrable in $D$ with the standard inner product.

We define the group of unitary operators $\left\{U^{t}\right\}, t \in \mathbb{R}$, in $L^{2} H(D)$ as follows:

$$
U^{t}(f)(z)=f(z+t)
$$

for $f \in L^{2} H(D)$ and $z \in D$. This is called the group of translation operators. The aim of this paper is to present some of its spectral properties.

We shall use the following notations.

Let $\mathcal{B}$ be the $\sigma$-algebra of Borel subsets of $\mathbb{R}$ and let $m$ denote the Lebesgue measure on $\mathcal{B}$. If $m_{j}, j=1,2$, are two measures on $\mathcal{B}$, we write $m_{1} \ll m_{2}$ to mean that $m_{1}$ is absolutely continuous with respect to $m_{2}$. Let $\widehat{f}$ denote the Fourier transform of $f \in L^{2}(\mathbb{R})([6]$, p. 17$)$.

For $t \in \mathbb{R}$ we define

$$
w(t)=\int_{J} e^{-4 \pi t y} d y
$$

and let $B_{J}=\{t \in \mathbb{R}: w(t)<\infty\}$. An easy computation shows that we have three possibilities: $B_{J}=\mathbb{R}, B_{J}=\mathbb{R}^{+}$or $B_{J}=\mathbb{R}^{-}$.

Let $L^{2}(\mathbb{R}, w)$ be the Hilbert space of all measurable functions $g$ on $\mathbb{R}$

1991 Mathematics Subject Classification: 47D10 
such that

$$
\int_{\mathbb{R}}\left|g(t)^{2}\right| w(t) d t<\infty .
$$

It is easy to see that for $g \in L^{2}(\mathbb{R}, w), g(t)=0$ for $m$-a.e. $t \notin B_{J}$.

We recall briefly the definition of the Genchev transform (see [2], [5] for more details).

For $f \in L^{2} H(D)$ and for $y \in J$ we set $f_{y}(x)=f(x+i y), x \in \mathbb{R}$. Then $f_{y} \in L^{2}(\mathbb{R})$ and we define the Genchev transform $G$ as follows:

$$
G(f)(t)=e^{2 \pi t y} \widehat{f}_{y}(t) .
$$

Note that the right side of (2) does not depend on $y \in J$.

The following theorem follows from the results of [2] and [5].

THEOREM 1. The Genchev transform is a unitary mapping of $L^{2} H(D)$ onto $L^{2}(\mathbb{R}, w)$. Moreover, for $g \in L^{2}(\mathbb{R}, w)$ and for $z \in D$ the function $t \mapsto e^{2 \pi i t z} g(t)$ belongs to $L^{1}(\mathbb{R})$ and the inverse of the Genchev transform has the form

$$
G^{-1}(g)(z)=\int_{\mathbb{R}} e^{2 \pi i t z} g(t) d t .
$$

We define the group of unitary operators $\left\{V^{t}\right\}, t \in \mathbb{R}$, in $L^{2}(\mathbb{R}, w)$ by

$$
V^{t}(g)(s)=e^{2 \pi i s t} g(s)
$$

for $g \in L^{2}(\mathbb{R}, w)$. The fundamental property of the Fourier transform gives

Theorem 2. $G \circ U^{t}=V^{t} \circ G$ for all $t \in \mathbb{R}$.

This shows that the groups $\left\{U^{t}\right\}$ and $\left\{V^{t}\right\}$ have the same spectral properties. Therefore we shall investigate $\left\{V^{t}\right\}$.

TheOREm 3. The projection-valued measure $P$ for the group $\left\{V^{t}\right\}$ is

$$
P(E) g=\chi_{(2 \pi)^{-1} E} g
$$

for $E \in \mathcal{B}$ and $g \in L^{2}(\mathbb{R}, w)$, where $\chi_{A}$ denotes the characteristic function of the set $A$.

Proof. It is easy to see that (5) defines a projection-valued measure on $\mathcal{B}([3]$, p. 12). by

For $g_{k} \in L^{2}(\mathbb{R}, w), k=1,2$, we define the finite complex measure $m_{g_{1} g_{2}}$

$$
m_{g_{1} g_{2}}(e)=\left\langle P(E) g_{1}, g_{2}\right\rangle
$$


for $E \in \mathcal{B}$. The definition of the inner product in $L^{2}(\mathbb{R}, w)$ and a change of variables gives

$$
m_{g_{1} g_{2}}(E)=\int_{E} \frac{1}{2 \pi} g_{1}\left(\frac{s}{2 \pi}\right) \overline{g_{2}\left(\frac{s}{2 \pi}\right)} w\left(\frac{s}{2 \pi}\right) d s
$$

for $E \in \mathcal{B}$ and from (4) we have

$$
\left\langle V^{t} g_{1}, g_{2}\right\rangle=\int_{\mathbb{R}} e^{2 \pi i s t} g_{1}(s) \overline{g_{2}(s)} w(s) d s=\int_{\mathbb{R}} e^{i u t} d m_{g_{1} g_{2}}(u) .
$$

Thus our statement follows from the spectral theorem ([3], p 23).

For $g \in L^{2}(\mathbb{R}, w)$ we denote by $C(g)$ the smallest closed subspace of $L^{2}(\mathbb{R}, w)$ containing $V^{t} g$ for all $t \in \mathbb{R}$. Moreover, let $m_{g}=m_{g g}$ (see (6)) and set $M(g)=\left\{h \in L^{2}(\mathbb{R}, w): m_{h} \ll m_{g}\right\}$.

Remark 1. It is easy to show that $M(g)$ is a closed subspace of $L^{2}(\mathbb{R}, w)$ and $C(g) \subset M(g)$.

Definition. The measure $m_{g}$ is called a maximal spectral type for $P$ if $m_{h} \ll m_{g}$ for every $h \in L^{2}(\mathbb{R}, w)$ (see also [1], p. 913).

LEMma 1. For $g \in L^{2}(\mathbb{R}, w)$ the following statements are equivalent:

(a) $g(t) \neq 0$ for $m$-a.e. $t \in B_{j}$

(b) $C(g)=L^{2}(\mathbb{R}, w)$,

(c) $m_{g}$ is a maximal spectral type for $P$.

Proof. (a) $\Rightarrow$ (b). Suppose that there exists $h \in L^{2}(\mathbb{R}, w)$ such that $h$ is orthogonal to $C(g)$. Then for all $t \in \mathbb{R}$

$$
0=\left\langle V^{t} g, h\right\rangle=\int_{\mathbb{R}} e^{2 \pi i t s} g(s) \overline{h(s)} w(s) d s .
$$

The function $g_{1}(s)=g(s) \overline{h(s)} w(s)$ belongs to $L^{1}(\mathbb{R})$ and $(7)$ shows that its Fourier transform is 0 . Thus $g_{1}(s)=0$ for $m$-a.e. $s \in \mathbb{R}$ and we conclude that $h(s)=0$ for $m$-a.e. $s \in \mathbb{R}$.

(b) $\Rightarrow$ (c) follows from Remark 1.

(c) $\Rightarrow\left(\right.$ a). Let $A=\left\{t \in B_{j}: g(t)=0\right\}$. Let $E$ be any Borel subset of $A$ such that $m(E)$ is finite. Then the function $h(t)=\chi_{E}(t)(w(t))^{-1 / 2}, t \in \mathbb{R}$, belongs to $L^{2}(\mathbb{R}, w)$. An immediate calculation shows that $m_{h}((2 \pi) E)=$ $m(E)$ and $m_{g}((2 \pi) E)=0$. Then (c) yields $m(E)=0$ and we conclude that $m(A)=0$.

Remark 2. The function $g(t)=e^{-t^{2}}(w(t))^{-1 / 2}, t \in \mathbb{R}$, belongs to $L^{2}(\mathbb{R}, w)$ and satisfies the condition (a) in Lemma 1 .

We notice that $m_{g} \ll m$ for all $g \in L^{2}(\mathbb{R}, w)$. Thus from Lemma 1 and Remark 2 we obtain 
COROllary 1. The group $\left\{V^{t}\right\}$ has continuous simple spectrum (for definition see [3], p. 21).

Remark 3. The simple spectrum of $\left\{V^{t}\right\}$ implies that the set of spectral types for $\left\{V^{t}\right\}$ has only one element ([1], p. 916).

Now we are going to prove some spectral properties of the group $\left\{U^{t}\right\}$ in $L^{2} H(D)$. Let $K_{D}(z, w)(z, w \in D)$ denote the Bergman function of the domain $D([4]$, p. 332$)$.

TheOREM 4. $1^{\text {O }}$ The projection-valued measure $Q$ for the group $\left\{U^{t}\right\}$ is

$$
Q(E)=G^{-1} P(E) G
$$

for $E \in \mathcal{B}$.

$2^{\mathrm{O}}$ The group $\left\{U^{t}\right\}$ has continuous simple spectrum.

$3^{\circ}$ For fixed $y \in J$ let $f(z)=K_{D}(z, i y)$ for $z \in D$. Then $C(f)=L^{2} H(D)$ and $m_{f}$ is a maximal spectral type for $Q$.

Proof. $1^{\circ}$ and $2^{\circ}$ follow immediately from Theorem 2 and Corollary 1.

$3^{\circ}$ Suppose that there exists $h \in L^{2} H(D)$ such that $h$ is orthogonal to $C(f)$. Then for all $t \in \mathbb{R}$ we have

$$
\left\langle h, U^{t} f\right\rangle=0 .
$$

Since $K_{D}(\cdot, w)$ has the reproducing property and $U^{t}$ is a unitary operator, from (9) and (1) we obtain

$$
0=\left\langle U^{-t} h, f\right\rangle=U^{-t} h(i y)=h(-t+i y) .
$$

This implies that $G(h)=0$ and by Theorem 1 we conclude that $h=0$. Hence $C(f)=L^{2} H(D)$ and Remark 1 shows that $m_{f}$ is a maximal spectral type for $Q$.

The projection-valued measure for the group $\left\{U^{t}\right\}$ has the following property:

TheOREM 5. For $E \in \mathcal{B}$ such that $m(E)$ is finite, for every $f \in L^{2} H(D)$ and every $z \in D$,

$$
Q(E)(f)(z)=\int_{\mathbb{R}} f(z+t) \widehat{\chi}_{(2 \pi)^{-1} E}(t) d t
$$

where the integrand belongs to $L^{1}(\mathbb{R})$.

Proof. For fixed $E \in \mathcal{B}$ let

$$
g(t)=\chi_{(2 \pi)^{-1} E}(t)
$$

Assume that $m(E)$ is finite. Then $g$ and $\widehat{g}$ belong to $L^{2}(\mathbb{R})$.

Let $z=x+i y \in D$. Then the function $f(z+t) \cdot \widehat{g}(t)=f_{y}(x+t) \widehat{g}(t)$, $t \in \mathbb{R}$, belongs to $L^{1}(\mathbb{R})$. 
From (8), (5), (3) and (2) we obtain

$$
\begin{aligned}
Q(E)(f)(z) & =G^{-1} P(E) G(f)(z)=\int_{\mathbb{R}} e^{2 \pi i t z} g(t) e^{2 \pi t y} \widehat{f}_{y}(t) d t \\
& =\int_{\mathbb{R}} e^{2 \pi i t x} \widehat{f}_{y}(t) g(t) d t .
\end{aligned}
$$

It is easy to see that the function $e^{2 \pi i t x} \widehat{f}_{y}(t)$ is the Fourier transform of $h(t)=f_{y}(t+x)$. Applying the multiplication formula for $h$ and $g$ ([6], p. 17) we obtain

$$
Q(E)(f)(z)=\int_{\mathbb{R}} \widehat{h}(t) g(t) d t=\int_{\mathbb{R}} h(t) \widehat{g}(t) d t=\int_{\mathbb{R}} f_{y}(t+x) \widehat{g}(t) d t,
$$

which completes the proof.

Remark 4. For every bounded interval $E=(a, b) \subset \mathbb{R},(10)$ has the form

$$
Q(E)(f)(z)=\int_{\mathbb{R}} f(z+t) \frac{e^{-i a t}-e^{-i b t}}{2 \pi i t} d t
$$

for $f \in L^{2} H(D)$ and $z \in D$.

Acknowledgment. The author is greatly indebted to Professor M. Skwarczyński for suggesting the problem.

\section{References}

[1] N. Dunford and J. T. Schwartz, Linear Operators, Vol. 2, Interscience Publishers, New York 1963.

[2] T. G. Genchev, Integral representations for functions holomorphic in tube domain, C. R. Acad. Bulgar. Sci. 37 (1984), 717-720.

[3] H. Helson, The Spectral Theorem, Lecture Notes in Math. 1227, Springer, Berlin 1986.

[4] E. Hille, Analytic Function Theory, Vol. 2, Ginn, Boston 1962.

[5] M. Skwarczyński, Alternating projections between a strip and a halfplane, Math. Proc. Cambridge Philos. Soc. 102 (1987), 121-129.

[6] E. Stein and G. Weiss, Introduction to Fourier Analysis on Euclidean Spaces, Princeton Univ. Press, 1971.

DEPARTMENT OF APPLIED MATHEMATICS

AGRICULTURAL ACADEMY

NOWOURSYNOWSKA 166

02-766 WARSZAWA, POLAND 